\title{
Egronomic Fingerprint Scanner Design for People with Motor Neuron Diseases
}

\author{
Abdulkareem Al-Alwani and Majdi Beseiso \\ Department of Computer Science and Engineering, Yanbu University College, Yanbu, Saudi Arabia
}

Received 2013-09-24, Revised 2013-10-02; Accepted 2013-10-28

\begin{abstract}
Fingerprint devices have evolved with time for authentication and identification purposes. It is used in generic security and social applications where identification and logging is required when entering that premises. In some circumstances the lag time increases due to increase in human entrees such as at immigration points, airports, random security checkups, attendance loggers. The increase in overall time due to individual human delay factors present a major hindrance in smooth security as well as organizational operations. The delay could occur due to non-technical factor such as not placing the fingers firmly in the surface of the device. This is a major cause of concern for senior citizens and people with motor neuron diseases such as Parkinson's, Huntington's and Alzheimer's disease. Therefore, a design is proposed in this research which can help the scanner to acquire fast and precise fingerprint scan of senior citizens and people with motor neuron diseases. This design uses ergonomically designed cover head for the scanner whose working is based on the Poka Yoke principle which assists firm finger placement on the scanner. In this research, 250 fingerprint scans were taken for statistical analysis using a normal fingerprint scanner and our proposed model scanner. Statistical comparison between the two results shows that our proposed model performs much better in terms of time consumption and accuracy.
\end{abstract}

Keywords: Fingerprint Device, Fingerprint Design, Biometric Authentication, Parkinson, Motor Neuron Disease

\section{INTRODUCTION}

Fingerprint scanning is a biometric method for user identification that is extensively used in organization and security applications. There are a multitude of other tests available which can be used for human identification based on unique features of a human body. Some methods scan iris or retina pattern on a human eye. Other methods test the facial features and identify a person based on that. Voice recognition is also used for identification in some cases. In addition, behavioral characteristics of human beings are also used to ascertain identification. For instance, it is common to check the keystroke, handwriting, signature recognition as methods of verification. Implementation of these methods in practical devices is subjected to many factors such as usability, familiarity or popularity, accuracy, cost and timing. Time factor is important attribute when the identification technology is realized for a large application and will be used to authenticate large number of people in fairly small amount of time. If the process cannot be done in a reasonable amount of time, then the technology can be considered unfeasible (Corcoran, 2013).

The time factor depends on two main features i.e., technical and non-technical. The technical feature is the computational ability of hardware and inherent efficiency of an algorithm to process the information extracted from a user and compare it against the template stored. This process significantly varies operational time for identification depending upon the type of hardware and algorithm used (Indi and Raut, 2013). There is a lot of published work on improving scanning time and accuracy using efficicient algorithms. In a case where large and diverse data is present, authentication can be accelerated using Corresponding Author: Abdulkareem Al-Alwani, Department of Computer Science and Engineering, Yanbu University College, Yanbu, Saudi Arabia 
intuitive feature selection. Two biometirc features measured at the same time have also shown improvement in time efficiency and accuracy (Basha et al., 2011). RGB color signature based fingerprint classification has shown promise in improving the lag time of normal scanning operations specially in color dense environments (Alsmadi et al., 2011). But increasing the computational power or algorithm aptness does not cater for random human errors, specifically when they're coming from people with motor neuron diseases.

The non-technical aspect is related to user's awareness and their capability of interacting with the device in a good timing manner. This aspect can considerably impact the time spent especially in the verification process where usually users have no helper at this point (Subramanian and Anand, 2013). For senior citizens and people with motor neuron diseases this aspect can contribute in increasing the time required for verification. An operator can help each subject individually which makes the presence of the operator vital at this stage. The usability of the device for our targeted group can be gained by increasing the awareness of the device that is used. Training on this device might be conducted in order to increase the familiarity level of this group towards the device. This, however, cannot be always accomplished in most of the situations as operator assistance is seldom used.

The technical aspect in lieu of the current technology is fully matured. The non-technical timing factor can be improved if the awareness of the device is increased using an innovative design. Our targeted group will not spend time in understanding how the device is functioning. A study (Abdul-Kareem and Yasser, 2012) suggests that the design of the device should conform to the physical attributes of the user in order to improve the familiarity and the ease of use. According to this study, such an improvement will minimize the time taken for every person (specifically from our targeted group) to extract his/her finger image. In addition, it will rectify errors that are not related to false rejection rate. Such errors usually occur due to the false positioning of the fingers on the device which leads to false reading. Also, the study suggests that this fault might occur due to incorrect scan of the finger caused due to improper finger placement.

This research will build upon the conclusion of previously mentioned study (Abdul-Kareem and Yasser, 2012). In this research an interface model for a fingerprint scanner is proposed that will improve the overall performance of the device. Moreover, this ergonomic design will help the targeted group to firmly place their fingers on device surfaces without a help of the operator thus reducing the overall identification time.

In the coming discussion, section II will discuss about design and specifications of our proposed model for a fingerprint scanner followed by section III and IV comprising of the comparative analysis and results. The conclusion and suggestions for the future work is presented in section $\mathrm{V}$.

\subsection{Design and Specifications}

The fingerprint scanner model proposed in this research is primarily designed for a specific group comprising of senior citizens, people with motor neuron diseases and people with disabilities. The main problem occurring with this group is improper placement of fingers on fingerprint scanners due to neurological and physical disabilities. The inception of this model is based upon the requirement of an ergonomic design (Privitera et al., 2009) sought out to assist our targeted group for prompt fingerprint identification in a short time thus improving the overall identification time of the device.

A poka yoke (Saurin et al., 2012) method is employed while designing the device which is an approach in a manufacturing process that helps an equipment operator avoids (yokeru) mistakes (poka). The primary advantage in following this mechanism is the identification and elimination of human generated errors by preventing, correcting, or drawing attention to the cause of their occurrence which in our case is improper placement of fingers. Overall, this particular design accounts for major physical constraints related to our targeted group, conformed into an ergonomic process to prevent incorrect operation by the user.

The fingerprint scanning fixture module in our research is designed, enabling the finger orientation and placement on the scanning area using a tunnel plastic cover shape which gently force the user finger into place for the scanner. The plastic cover has a tunnel shape which will control the movement of the finger to the scanning area using carefully designed structural ergonomics. The tunnel is designed to accommodate finger shape appropriately for scanning action without exerting too much effort. The concept model is shown in Fig. 1.

ZK4000 fingerprint scanner is shown in Fig. 2. The scanner is mounted on an Artilon $7 \mathrm{~mm}$ base plate to allow minimum vibrations on finger or hand contact. Scanner is then enclosed in a plastic fixture with an aperture opening, attaching it to a rotating tunnel head as shown in Fig. 3. 


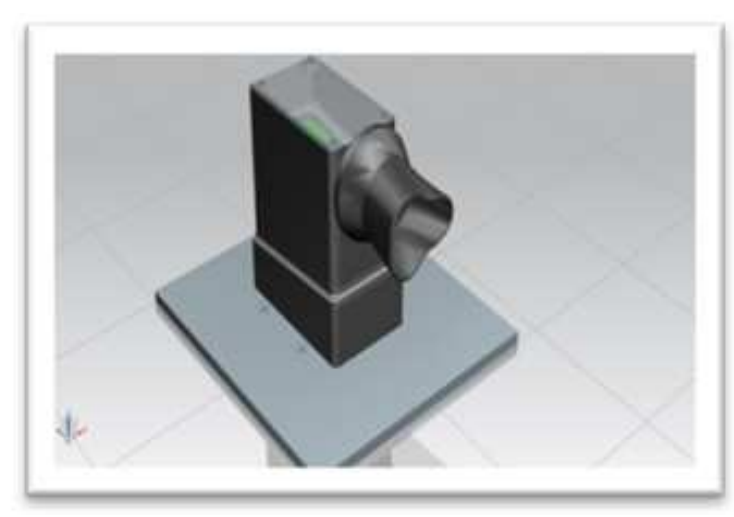

Fig. 1. Rotating head design to control tunnel aperture

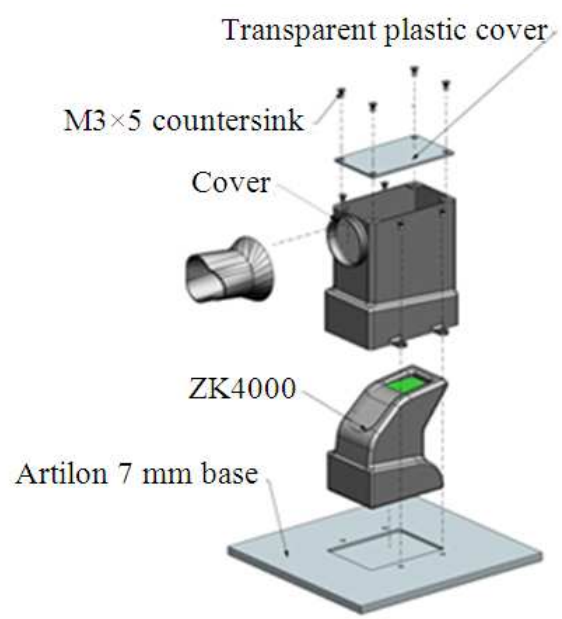

Fig. 2. Ergonomic fixture design employing ZK4000 Fingerprint scanner

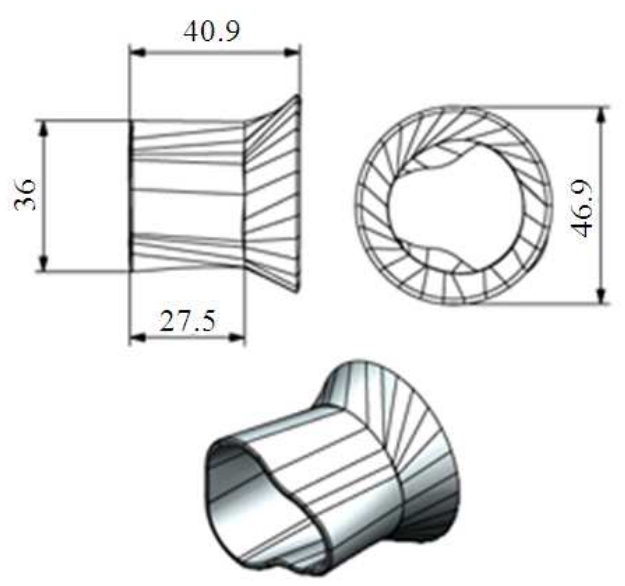

Fig. 3. Rotating head design to control tunnel aperture

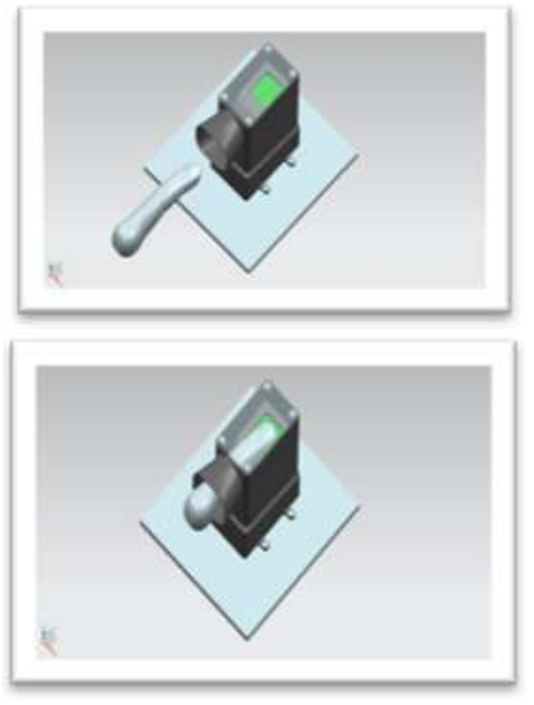

Fig. 4. Unidirectional movement of the finger on a single axis in the new model: Finger out and inserting finger for scanning on a single axis

It can be seen in the figure that model is adjustable to the finger and the tunnel aperture can be adjusted with rotating head. Top of the fixture is made of transparent plastic to allow for visual observations. The cover head is designed to optimize the scanning process by catering the finger attributes which affect the fingerprint impression.

A Rotating head shown in Fig. 3 is interfaced with the tunnel that accommodates individual size of all five fingers and can be rotated to increase or decrease the aperture of the tunnel. The head function also allows directing the finger to the right position on the scanner which increases the scanning time and accuracy. This rotating head plays a vital role in improving the scan time and false rejection rate. The new rotating head accommodates all fingers in the scan process as it conforms to the shape of any finger inserted for a scan by simple rotation of the head. By this innovation, finger head can be snapped in on the cover body for an accurate scan.

This approach for fingerprint scanning provides us with following advantages:

- Simple and flexible design

- Cost effective

- Minimal time delay

- Ergonomic based

- Accurate readings 
Overall, ergonomics design of the tunnel facilitates natural pressing action of the finger on the scan area while 'poka yoke' approach in the tunnel design restrains the finger movement on a single axis as shown in Fig. 4. In the coming section testing methodology for the new model will be discussed.

\section{MATERIALS AND METHODS}

A total of around 50 participants were approached for fingerprint scanning which included 20 subjects with motor neuron diseases with the rest of the group belonging to age group greater than 60 . The choice to participation was kept strictly voluntary. Two hospitals and three shopping marts were chosen for collection of sample scans. All five fingers of each subject's hand were individually scanned twice making the total amount of sample fingerprint images to 500. The first 250 fingerprint samples were taken using a normal scanner. Rest 250 samples were collected using the improved version of this scanner i.e., with tunnel plastic cover. The two samples of 250 scans each are the compared to assess the scanning time and reading variations between the two set of samples.

The fingerprint scans in sample 1 is designated with following names: Thumb (T1), index (I1), middle1 (M1), ring1 (R1) and babyl (B1) while the same fingers in sample no 2 is designated as: T2, I2, M2, R2 and B2. The results are presented in the following section.

\section{RESULTS}

This section will present comparative results using ergonomic tunnel based model and normal fingerprint scanner. The data labeled T1, I1, M1, R1 and B1 refer to the scan time readings without the model. The data labeled T2, I2, M2, R2 and B2 refer to the scan time readings with the model. Table 1 shows the means of the reading of all fingers with and without the model.

Detailed statistical results pertaining to scanning time of every individual finger with normal and improved scanner are given in Table 2 and 3 respectively. The minimum mean value among all the fingers is exhibited by thumb in both the models. Additionally, the minimum reading of the thumb 1 and 2 was also lowest as compared to other fingers. This is because more strength is involuntary applied when a human thumb is pressed against a hard surface. This causes a better scan result in a shorter amount of time and a low percentage of false rejection rates. This also ascertains the notion that more strength applied by a finger in scanning provides a qualitative in reading accuracy.

The baby fingers have the largest means and medians comparing to other fingers as well as minimum and maximum. This also supports the previous claim; more strength leads to better readability and vice versa. Therefore, the new model will help to give more support and strength to the weakest fingers in order to increase its quality. The range of reading is varied from fingers to another.

Table 1. Mean scan time of Normal and ergonomic scanner

\begin{tabular}{llll}
\hline $\begin{array}{l}\text { Normal } \\
\text { scanner }\end{array}$ & $\begin{array}{l}\text { Mean time } \\
(\mathrm{sec})\end{array}$ & $\begin{array}{l}\text { Ergonomic } \\
\text { scanner }\end{array}$ & $\begin{array}{l}\text { Mean time } \\
(\mathrm{sec})\end{array}$ \\
\hline Thumb1 & 4.10 & Thumb2 & 3.85 \\
Index1 & 4.52 & Index2 & 4.10 \\
Middle1 & 4.30 & Middle2 & 3.94 \\
Ring1 & 4.66 & Ring2 & 4.12 \\
Baby1 & 5.12 & Baby2 & 4.20 \\
\hline
\end{tabular}

Table 2. Statistical results for scan time samples from normal

\begin{tabular}{|c|c|c|c|}
\hline Fingers & Statistical indicator & Values (sec) & False scan rate $(\%)$ \\
\hline & Mean & 4.10 & 10 \\
\hline \multirow{5}{*}{ Thumb1 } & Median & 4.15 & \\
\hline & Std.Dev & 0.30 & \\
\hline & Minimum & 3.98 & \\
\hline & Maximum & 4.15 & \\
\hline & Range & 0.17 & \\
\hline \multirow[t]{6}{*}{ Index 1} & Mean & 4.52 & 20 \\
\hline & Median & 4.48 & \\
\hline & Std.Dev & 0.50 & \\
\hline & Minimum & 4.40 & \\
\hline & Maximum & 4.60 & \\
\hline & Range & 0.20 & \\
\hline \multirow[t]{6}{*}{ Middle1 } & Mean & 4.30 & 16 \\
\hline & Median & 4.28 & \\
\hline & Std.Dev & 0.60 & \\
\hline & Minimum & 4.16 & \\
\hline & Maximum & 4.38 & \\
\hline & Range & 0.22 & \\
\hline \multirow[t]{6}{*}{ Ring1 } & Mean & 4.66 & 21 \\
\hline & Median & 4.60 & \\
\hline & Std.Dev & 0.55 & \\
\hline & Minimum & 4.50 & \\
\hline & Maximum & 4.80 & \\
\hline & Range & 0.30 & \\
\hline \multirow[t]{6}{*}{ Baby1 } & Mean & 5.12 & 30 \\
\hline & Median & 5.09 & \\
\hline & Std.Dev & 0.80 & \\
\hline & Minimum & 4.95 & \\
\hline & Maximum & 5.21 & \\
\hline & Range & 0.26 & \\
\hline
\end{tabular}


Table 3. Statistical results for scan time samples from ergonomic scanner

\begin{tabular}{|c|c|c|c|}
\hline Fingers & Statistical indicator & Values (sec) & False scan rate $(\%)$ \\
\hline \multirow{6}{*}{ Thumb2 } & Mean & 3.85 & 2 \\
\hline & Median & 3.83 & \\
\hline & Std.Dev & 0.25 & \\
\hline & Minimum & 3.79 & \\
\hline & Maximum & 3.90 & \\
\hline & Range & 0.15 & \\
\hline \multirow{6}{*}{ Index2 } & Mean & 4.10 & 3 \\
\hline & Median & 4.12 & \\
\hline & Std.Dev & 0.50 & \\
\hline & Minimum & 4.01 & \\
\hline & Maximum & 4.20 & \\
\hline & Range & 0.19 & \\
\hline \multirow[t]{6}{*}{ Middle2 } & Mean & 3.94 & 4 \\
\hline & Median & 3.90 & \\
\hline & Std.Dev & 0.45 & \\
\hline & Minimum & 3.85 & \\
\hline & Maximum & 4.03 & \\
\hline & Range & 0.18 & \\
\hline \multirow{6}{*}{ Ring2 } & Mean & 4.12 & 3.5 \\
\hline & Median & 4.14 & \\
\hline & Std.Dev & 0.52 & \\
\hline & Minimum & 4.05 & \\
\hline & Maximum & 4.23 & \\
\hline & Range & 0.18 & \\
\hline \multirow[t]{6}{*}{ Baby2 } & Mean & 4.20 & 6 \\
\hline & Median & 4.16 & \\
\hline & Std.Dev & 0.55 & \\
\hline & Minimum & 4.10 & \\
\hline & Maximum & 4.30 & \\
\hline & Range & 0.20 & \\
\hline
\end{tabular}

However, the lowest range is seen in the thumb 1 and 2 because it has the lowest minimum value. In the baby fingers 1 and 2, the range is largest because the largest time delay value appeared with them.

\section{DISCUSSION}

To test the functionality of the new model for our target group, a sample of 50 individuals were selected for a scan test on a normal scanner and another one with our proposed ergonomic model. It was observed that there was an improvement in the reading after using the suggested model. Directing finger to the scan area surface via a rotatable tunnel aperture contributes to an accurate reading or a high quality fingerprint scan. This not only reduces the time to a first time scan but also the time to verify, thus reducing any unnecessary human error induced delays. In addition, the result indicates that there are remarkable improvement in the reading of the two small fingers (ring and baby fingers) from the old reading (without using the model) and the new reading (using the model). This improvement was due to the natural weakness of these two fingers. The design of the model gave better support for the baby, ring and other fingers.

Comparing our research with latest approaches, an approach proposed in a recently registered patent (Allen, 2012), was investigated for comparative analysis. This approach proposed a design consisting of a finger guide device for a biometric fingerprint scanner with a raised portion and an aperture formed through the device at the channel. Contours for finger selection are configured to indicate the finger to be inserted. This approach still doesn't cater for the elderly and people with motor neuron diseases as firm placement of fingers is essential for accurate fingerprint scan which cannot be made possible using finger shaped contours. Contours for fingers helps in positioning but not firm placement. In this research the rotatable head grips the finger so that there is no chance of vibration or free movement of finger. This promises a better scan time and accuracy during scanning.

\section{CONCLUSION}

This study introduces the reader with a unique approach of using an ergonomic design to improve the quality and lower the time delay for a normal fingerprint scan. The design was made with a view to accommodate senior citizens and people with motor neuron diseases. Mechanical outline of the design caters for the anatomy and motor function of human fingers based on automated ergonomic design approaches (Dass, 2010). This research further explains shortcomings of a normal fingerprint scanner and how it affects our target group in crowded areas such as airports and hospitals.

To sum up, this design improvement is necessary for fingerprint scanners to facilitate elderly and people with motor neuron diseases. This design approach not only improves the scanning ability of the device. In addition, it will help the people who are using the device for the first time to place their finger firmly and the proper place. Also, it will support the fingers to ensure proper scanning of the image until the finger is released from the tunnel.

The model proposed in this study has many advantages. It's cost effective and introduces improvement to scan accuracy for people with motor function problems. The design is mechanically self contained without any need of sensors and electric connections. More importantly, it has the feature of operational and physical compatibility with most of the commercially available fingerprint scanners due to 
its ergonomic nature. The main limitation involved in this device is that it is manually operated which can incur human errors affecting the scanning process. Such errors are rare but are inherent due to the manual nature of the proposed model. These errors can be removed by employing automation but it will increase the overall cost of the device. The final section will discuss the future work pertaining to the automation of the proposed model.

\subsection{Future Work}

In future work the model may be automated to perform without any operator/user interference with features accommodating shortcomings of our targeted group (Sauhats et al., 2011). This will remove remaining human errors involved due to manually assisted fingerprint scanning of our targeted group. This can be achieved using pressure sensors or a push button. When the device senses the fingers or the push button is pressed, the upper part of the device will move from the top to bottom in 90-degree way to grip the finger for firm placement. After having the fingers scanned, then the upper part can move back to its original place to release the fingers. Then, after the user removes his hand from the device, a wiper may clean the glass surface to clean up the previous imprint and to make it hygienic for the new user.

\section{REFERENCES}

Abdul-Kareem, A.A. and A. Yasser, 2012. Testing the familiarity and the ease of using Fingerprint devices and how the current design affecting the accuracy. Br. J. Arts Soc. Sci., 6: 51-51.

Allen, R., 2012. Ergonomic finger guide for biometric finger scanner.

Alsmadi, M.K., K.B. Omar and S.A.M. Noah, 2011. Fish classification based on robust features extraction from color signature using back-propagation classifier. J. Comput. Sci., 7: 52-58. DOI: $10.3844 /$ jessp. 2011.52 .58
Basha, A.J., V. Palanisamy and T. Purusothaman, 2011. Efficient multimodal biometric authentication using fast fingerprint verification and enhanced iris features. J. Comput. Sci., 7: 698-706. DOI: 10.3844/jcssp.2011.698.706

Corcoran, P.M., 2013. Biometrics and consumer electronics: A brave new world or the road to dystopia. IEEE Consumer Electron. Mag., 2: 22-33. DOI: 10.1109/MCE.2013.2239152

Dass, S.C., 2010. Assessing fingerprint individuality in presence of noisy minutiae. IEEE Trans. Inform. Forensics Security, 5: 62-70. DOI: 10.1109/TIFS.2009.2039598

Indi, T.S. and S.D. Raut, 2013. Person identification based on multi-biometric characteristics. Proceedings of the International Conference on Emerging Trends in Computing, Communication and Nanotechnology, Mar. 25-26, IEEE Xplore Press, Tirunelveli, pp: 45-52. DOI: 10.1109/ICE-CCN.2013.6528611

Privitera, M.B., M. Design and D.L. Murray, 2009. Applied ergonomics: Determining user needs in medical device design. Proceedings of the Annual International Conference on IEEE Engineering in Medicine and Biology Society, Sept. 3-6, IEEE Xploe Press, Minneapolis, MN., pp: 5606-5608. DOI: 10.1109/IEMBS.2009.5333781

Sauhats, A., J. Kucajevs, L. Leite, G. Bockarjova and A. Utans, 2011. Out-of-step automation device model validation methodology. Proceedings of the 10th International Conference on Environment and Electrical Engineering, May 8-11, IEEE Xplore Press, Rome, pp: 1-6. DOI: 10.1109/EEEIC.2011.5874606

Saurin, T.A., J.L.D. Ribeiro and G. Vidor, 2012. A framework for assessing poka-yoke devices. J. Manufactur. Syst., 31: 358-366. DOI: 10.1016/j.jmsy.2012.04.001

Subramanian, M.S. and S. Anand, 2013. Fuzzy system to authenticate assisted living patients in their home environment. J. Comput. Sci., 9: 558-565. DOI: 10.3844/jessp.2013.558.565 